\title{
Synthesis and Evaluation of Zeolite Surface-Modified Perlite
}

\author{
Makoto Kasai ${ }^{1}$, a , Yosei Kobayashi ${ }^{1}$, Masakazu Togo ${ }^{2}$ and Atsushi Nakahira ${ }^{2,3}$ \\ 1 Perlite Division, Mitsui Mining \& Smelting Co., Ltd., Japan \\ ${ }^{2}$ Department of Materials Science Biomaterial Group, Osaka Prefecture University, Japan \\ ${ }^{3}$ Trans-Regional Corporation Center for Industrial Materials Research, IMR, Tohoku University, Japan
}

\begin{abstract}
Perlite is volcanic glass mainly composed of amorphous aluminum silicate, mainly composed $\mathrm{SiO}_{2}$ and $\mathrm{Al}_{2} \mathrm{O}_{3}$ with less impurities such as heavy metals. Amorphous (glassy) perlite is used in lightweight aggregate and insulation. In addition, it has also been used as a filter aid by grinding the expanded perlite. However, it has not been used as environmental cleanup materials, because the ion exchange capacity of the perlite is very low. In this study, we tried to synthesize the hybrid filter aid with chemical adsorption capacity by synthesizing the zeolite on the surface of the perlite. As a result, by using the hydrothermal synthesis method, zeolite surface modified perlite was synthesized in which the LTA type zeolites were generated on the surface of the perlite.
\end{abstract}

\section{Background}

Perlite is an aluminum silicate mainly composed of perlite ore. This perlite (expanded perlite ore) has porous structure. So it is a lightweight glass material with superior physical properties, such as an excellent thermal, flame, and chemical resistance, as well as thermal insulation. In addition, it is used as a heat-insulating material in building materials and large tanks, such as LNG tanks, as well as soil improvement material.

Perlite is produced by crushing the raw material and then subjecting it to heat processing. As volcanic glass, such as perlite ore, contains the water in the glass structure, the water steam generates on the heattreatments ${ }^{[1],[2]}$. When the generation temperature of the gas is equal to or higher than the glass softening point, the glass is expanded with the generated gas. This means that by rapidly heating perlite ore using a rotary kiln, the volatile constituents (mainly water) of the raw material rapidly become volatile and expand when the glass softens.

Also, expanded perlite that has been crushed and classified can be used as a filter aid. Filter aids are additives used to increase the filtering performance for solid and liquid separation. Perlite is generally used in filter aid because this material is required to have a low bulk density, settleability, and must be incompressible with small cake specific resistance.

Separation of solids from liquids, on the other hand, can be accomplished using filtering equipment such as filter presses. At that time, a filter aid is added to improve the filtration efficiency. However, since the filter aid itself has no adsorption ability, it is necessary to add an adsorbent to remove contaminants such as heavy metals. Therefore, it was a problem to increase the amount of generated waste cake. In this regard, if adsorption capacity can be imparted to the perlite filter aid, it is possible to achieve excellent filtration efficiency without increasing the amount of waste cake produced. This has been considered a potential means to provide a hybrid purifying material.

Zeolite is a generic term of porous aluminosilicate, the basic unit is three-dimensional framework structures consisting of tetrahedrally coordinated $\mathrm{SiO}_{4}$ and $\mathrm{AlO}_{4}$ units. When a part of $\mathrm{Si}^{4+}$ is replaced with $\mathrm{Al}^{3+}$, lack of positive charge occurs, cation exchange capability of the zeolite is obtained by supplementing the shortage cation such as an alkali metal ${ }^{[3]}$.

Taking into consideration that perlite is a powder of aluminum silicate, it can be considered a raw material that can be used as a source of silicon ion and aluminium ion in zeolite synthesis. The focus of this research was to synthesize zeolite from perlite, especially to synthesize zeolite on the surface of perlite for filter aid. This latter material can then be used to synthesize a perlite filter aid with ion exchange and adsorption capacities to increase its value as an environmental purification material. In past studies, perlite ore and expanded perlite have been used as starting materials in an attempt to synthesize a variety of zeolites ${ }^{[4]-[8]}$. However, there has not been any report made on using perlite as a raw material to produce compounds with zeolites, while sustaining its characteristics. In this work, a perlite filter aid was fabricated with added chemical characteristics, such as adsorption and ion exchange capacities, both of which are not features of conventional filter aids.

\footnotetext{
${ }^{a}$ Corresponding author: m_kasai@mitsui-kinzoku.co.jp
} 


\section{Methods}

\subsection{Specimen}

The perlite filter aid samples used for the experiment were manufactured using perlite ore produced in Kitakata City of the Fukushima Prefecture. The perlite used was prepared by grinding and classifying the perlite ore, which was then heated with a rotary kiln, and lastly expanded and crushed to prepare the perlite filter aid. The derived perlite was analyzed for its chemical composition by using a fluorescent X-ray analysis system (XRF; model RIX-3000 manufactured by Rigaku Corporation). The particle diameter of the perlite used in this synthesis experiment was measured using laser diffraction and scattering particle diameter distribution measurement equipment (model MT3000 II manufactured by MicrotracBEL Corp.). Furthermore, the specific surface area of the perlite was measured using an automatic specific surface area measurement apparatus (model BELSORP-mini II manufactured by MicrotracBEL Corp.) and the BET method.

\subsection{Synthesis of zeolite}

For the filter aid, $0.70 \mathrm{~g}$ of perlite was collected in a 22 $\mathrm{ml}$ Teflon container. To adjust the $\mathrm{Si} / \mathrm{Al}$ ratio to 1.0 and 2.0, a prescribed quantity of sodium aluminate (Wako Pure Chemical Industries, Ltd.) was added there. Afterwards, $15 \mathrm{ml}$ of a $1.0 \mathrm{M} \mathrm{NaOH}$ solution was added to the container. The solution was mixed and aged at $25^{\circ} \mathrm{C}$ for 24 hours. An autoclave system was then used to perform the hydrothermal synthesis at $55^{\circ} \mathrm{C}$ for $72 \mathrm{~h}$.

The hydrothermal synthesis was also performed using a fixed silicon to aluminum ratio of 1.0, a synthesis temperature of $55^{\circ} \mathrm{C}$, and different reaction times of 72 and $96 \mathrm{~h}$. The derived product was filtered, then cleansed with distilled water, and lastly dried in an oven at $50{ }^{\circ} \mathrm{C}$ for $24 \mathrm{~h}$. The dried specimen was then crushed in an agate mortar to prepare the powder specimens.

\subsection{The heavy metal removal capability}

The heavy metal removal capability tests were done for the zeolite surface modified perlite and zeolite reagent. Each sample of $0.2 \mathrm{~g}$ was immersed for 1 to 12 hours to cadmium nitrate solution in each $20 \mathrm{ml}$ of $10 \mathrm{ppm}$. Then, the solutions were filtered and then the residual metal ion concentration were measured using the ICP emission spectral analysis (ICP; model PS7800 manufactured by Hitachi High-Tech Corporation) to evaluate the ion removing capacity.

\subsection{Characterization of the synthesized sample}

The structural analysis of the samples obtained was carried out using the powder X-ray diffraction method
(XRD; model Mini Flex II manufactured by Rigaku Corporation). The measurement range was set to fall between $2^{\circ}$ to $60^{\circ}$, at a scanning speed of $4 \% \mathrm{~min}$, a voltage of $30 \mathrm{kV}$, a current of $15 \mathrm{~mA}$, and a sampling width of $0.02^{\circ}$. Their structure and microstructures of obtained synthesis products were observed with a scanning electron microscope (SEM; model VE-8800 manufactured by Keyence).

Furthermore, the vapor adsorption isotherm of the derived specimens were measured using a high precision, fully automated, gas adsorption apparatus (model BELSORP-18 manufactured by MicrotracBEL Corp.); and the BET specific surface area was measured using the BET multi-point method.

The structural analysis of the detailed fine structures of the silicon and aluminum in the derived specimens was performed using X-ray adsorption fine structure analysis (XAFS), using the beam lines from two different radiation facilities (BL6N1 at Aichi Synchrotron Radiation Center and BL-10 at the SR Center of Ritsumeikan University). In both instances, the measurements were performed using the fluorometric method, with the measurements for the K-edge adsorption of silicon performed using BL6N1 at Aichi Synchrotron Radiation Center; and the measurements for the K-edge adsorption of aluminum performed with BL10 at the SR Center of Ritsumeikan University.

The structural analysis of the detailed fine structures of the cadmium in the heavy metal removal test samples were performed using X-ray adsorption fine structure analysis (XAFS), using BL14B2 at Spring-8. The measurements were performed using the fluorometric method, with the measurements for the K-edge adsorption of cadmium performed using BL14B2 at Spring-8.

\section{Results}

\subsection{Physical properties of perlite}

Table 1 shows the chemical composition of perlite. The perlite consisted of $75 \mathrm{wt} \% \mathrm{SiO}_{2}, 14 \mathrm{wt} \% \mathrm{Al}_{2} \mathrm{O}_{3}$ and, from the table, it is evident that $\mathrm{SiO}_{2}$ and $\mathrm{Al}_{2} \mathrm{O}_{3}$ comprised approximately $90 \%$ of the perlite. Furthermore, while $\mathrm{Na}_{2} \mathrm{O}, \mathrm{K}_{2} \mathrm{O}$, and $\mathrm{CaO}$ were also present, their contents were low. Similarly, the contents of other metal elements were also very small.

Table 1. Composition of perlite

\begin{tabular}{c|c|c|c|c|c}
\hline \multicolumn{7}{c}{ Chemical composition } \\
[wt\%] \\
\hline $\mathrm{SiO}_{2}$ & $\mathrm{Al}_{2} \mathrm{O}_{3}$ & $\mathrm{Fe}_{2} \mathrm{O}_{3}$ & $\mathrm{Na}_{2} \mathrm{O}$ & $\mathrm{K}_{2} \mathrm{O}$ & $\mathrm{CaO}$ \\
\hline 75.1 & 14.3 & 0.9 & 3.5 & 5.2 & 0.3 \\
\hline
\end{tabular}


Fig.1 shows the particle diameter distribution of the perlite used in the test. The median diameter (D50) of the perlite used in the synthesis was $30.1 \mu \mathrm{m}$, while its specific surface area (by BET method) was $3.2 \mathrm{~m}^{2} \cdot \mathrm{g}^{-1}$.

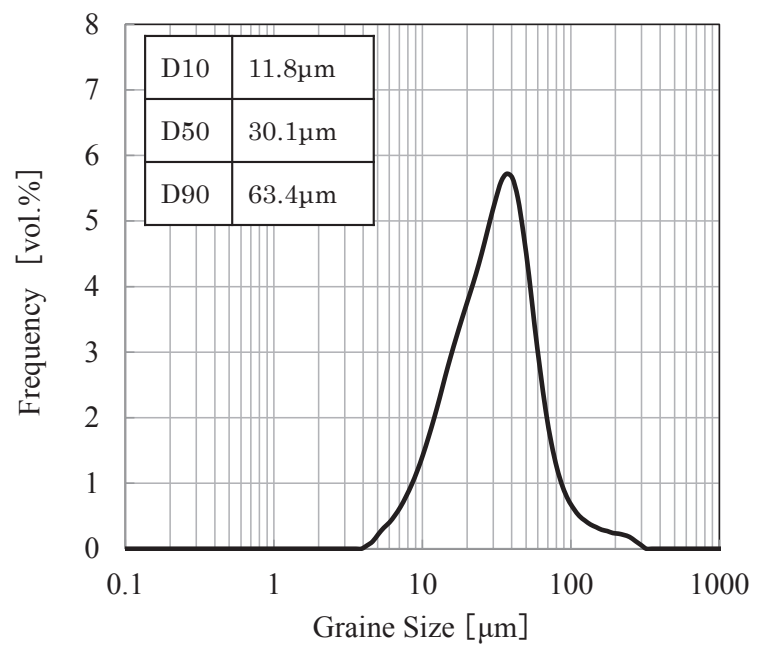

Fig. 1 Grain size distribution of perlite.

Furthermore, the XRD measurement results for perlite are shown in Fig. 2. The derived spectrum contained a halo peak, which confirmed that the perlite had an amorphous structure.

\subsection{Synthesis of zeolite using perlite}

Table 2 shows the different amounts of compounds used to prepare the various systems.

Table 2. Quantity of starting materials

\begin{tabular}{|c|c|c|c|c|c|c|c|c|}
\hline \multirow{2}{*}{$\begin{array}{c}\text { Perlite } \\
{[\mathrm{g}]}\end{array}$} & \multicolumn{2}{|c|}{$\mathrm{NaOH}$} & \multicolumn{4}{|c|}{ Chemical Composition ratio } & \multirow{2}{*}{$\mathrm{Si} / \mathrm{Al}$} & \multirow{2}{*}{$\mathrm{Na} / \mathrm{Si}$} \\
\hline & {$[\mathrm{mol} / \mathrm{L}]$} & [ml] & $\mathrm{SiO}_{2}$ & $\mathrm{Al}_{2} \mathrm{O}_{3}$ & $\mathrm{Na}_{2} \mathrm{O}$ & $\mathrm{H}_{2} \mathrm{O}$ & & \\
\hline 0.7 & 1.0 & 15 & 1.0 & 0.26 & 1.12 & 95.32 & 2.0 & 2.2 \\
\hline 0.7 & 1.0 & 15 & 1.0 & 0.53 & 1.51 & 95.32 & 1.0 & 3.0 \\
\hline
\end{tabular}

The silicon to aluminum ratio was controlled to be 1.0 to 2.0 using sodium aluminate.

Fig.2 shows the XRD results of the specimen obtained from the hydrothermal synthesis performed at $55{ }^{\circ} \mathrm{C}$. Fig. 3 shows the SEM images. The XRD results show that peaks attributed to LTA type zeolite. Furthermore, as the silicon to aluminum ratio became lower, the peak intensity attributed to the LTA zeolite became stronger. The broad peak derived from perlite remained between $15^{\circ}$ to $40^{\circ}$ degrees, indicating that the perlite had not been entirely converted into zeolite.

Results of the SEM observations confirmed that for samples between silicon to aluminum ratios ranging from 1.0 to 2.0 , the squamation particles of the perlite filter aid remained. Furthermore, precipitation of the threedimensional LTA type zeolite was confirmed on the surface and edges of the perlite filter aid. The amount of LTA type zeolite that precipitates increased as the silicon to aluminum ratio became lower, while the particle diameters of the precipitated LTA type zeolite were observed to become microscopic, decreasing from about $2 \mu \mathrm{m}$ to $0.5 \mu \mathrm{m}$. Furthermore, as the silicon to aluminum ratio became lower, the LTA type zeolite particles were observed to assume organized threedimensional shapes.

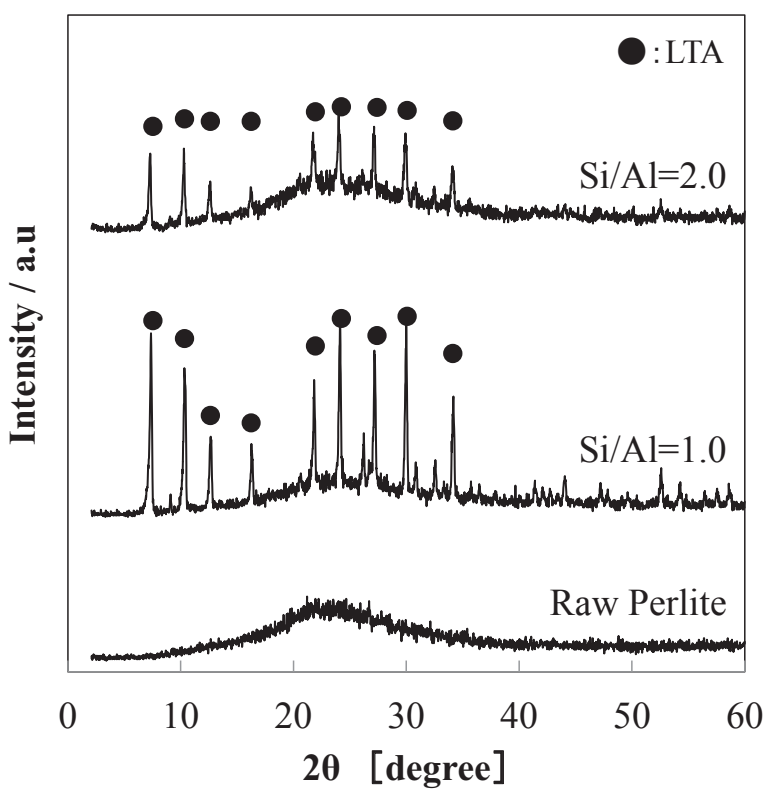

Fig.2 XRD patterns of the products obtained from the hydrothermal synthesis carried out at $55^{\circ} \mathrm{C}$.
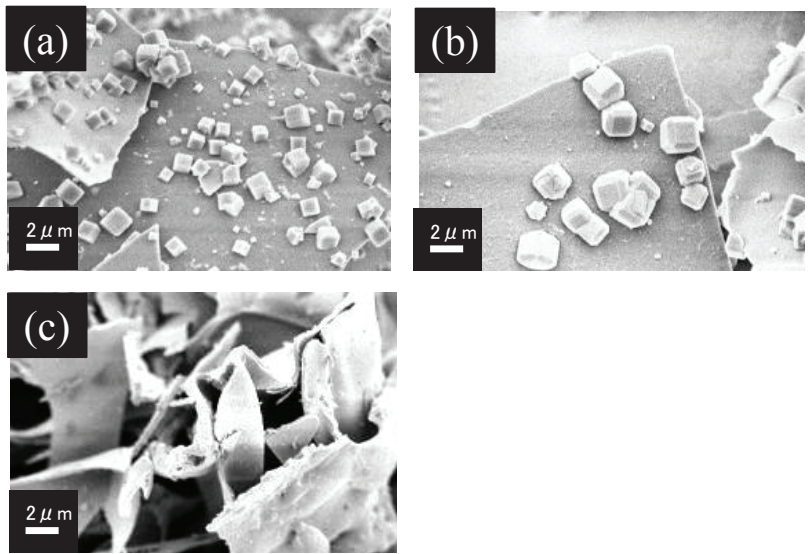

Fig.3 SEM images of the products obtained from the hydrothermal synthesis carried out $55^{\circ} \mathrm{C}$, and at different $\mathrm{Si} / \mathrm{Al}$ ratios (a: $1.0 ; \mathrm{b}: 2.0$; c: raw perlite).

Fig. 4 shows the XRD results of specimens prepared at reactions times of 72 and $96 \mathrm{~h}$, conducted with a silicon to aluminum ratio of 1.0 and at $55^{\circ} \mathrm{C}$. Fig. 5 shows their SEM images for the products. The XRD measurement results revealed that the intensity of peaks attributed to LTA type zeolite became stronger as the reaction time increased, and reached about one thrid peak intensity of the LTA type zeolite reagent (zeolite A-4 manufactured by Tosoh Corporation) when the reaction time was $96 \mathrm{~h}$. 


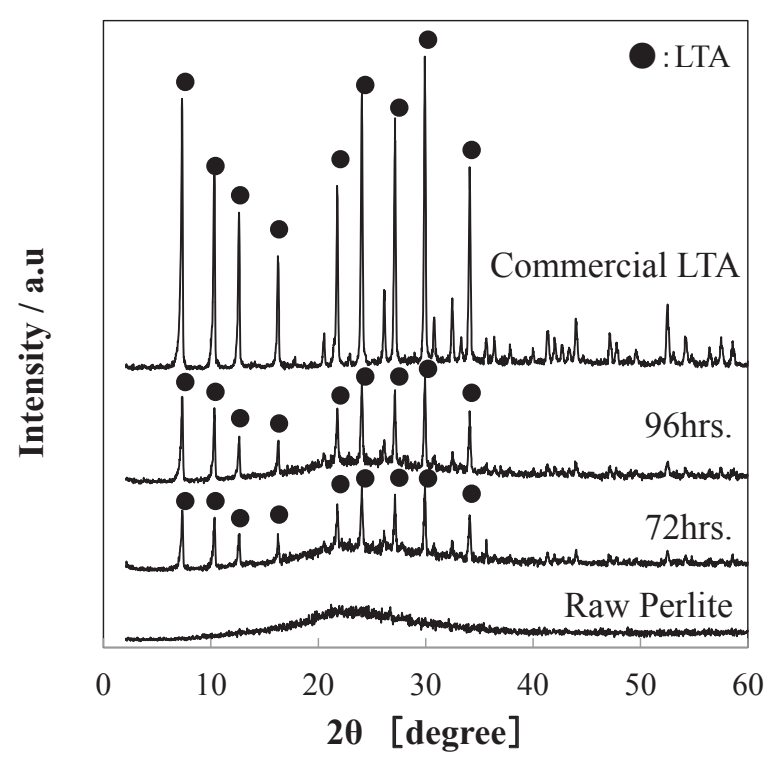

Fig.4 XRD patterns of the products from the hydrothermal synthesis carried out at $75^{\circ} \mathrm{C}$ for 72 and $96 \mathrm{~h}$.

A verification of the SEM images confirms that the number of LTA type zeolites generated on the surface of perlite increased as the synthesis time increased. The diameters of the generated LTA type zeolite particles were about 1 to $2 \mu \mathrm{m}$, even when the synthesis time was increased. For these samples, the generation of LTA type zeolite was minute, even when compared with the diameters of LTA type zeolite reagent particles (about 5 $\mu \mathrm{m})$.

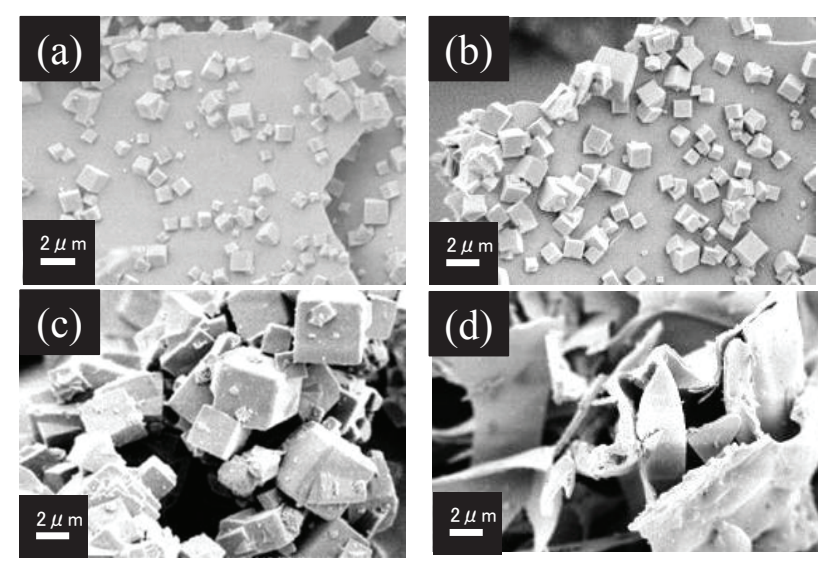

Fig.5 SEM images of the products obtained by carrying out the hydrothermal synthesis at different reaction times of (a) $72 \mathrm{~h}$, (b) $96 \mathrm{~h}$, (c) LTA zeolite reagent and (d) raw perlite.

Table 3 shows the BET specific surface area. It was calculated using the BET multi-point method on the vapor adsorption isothermal line. The BET specific surface area of the perlite filter aid, which was used as the raw material, was extremely small with a value of 3.2 $\mathrm{m}^{2} \cdot \mathrm{g}^{-1}$. However, this value increased to $194 \mathrm{~m}^{2} \cdot \mathrm{g}^{-1}$ after performing the hydrothermal synthesis for $72 \mathrm{~h}$. When the hydrothermal synthesis time increased to $96 \mathrm{~h}$, the specific surface area increased to $246 \mathrm{~m} 2 \cdot \mathrm{g}-1$, with the latter specific surface area reaching about one third the value of the specific surface area of the LTA type zeolite reagent. A correlation was observed between this increased specific surface area and the amount of precipitates on the LTA type zeolite generated on the surface of the perlite filter aid. In addition, the ratio of the BET specific surface area and the peak intensity ratio from the XRD results were in accordance with each other.

Table 3. Specific surface area of commercial LTA, perlite, and the products of the hydrothermal synthesis.

\begin{tabular}{cc}
\hline \multicolumn{2}{c}{ Specific surface are $/ \mathrm{m}^{2} \cdot \mathrm{g}^{-1}$} \\
\hline Commercial LTA & 624 \\
Perlite & 3.2 \\
Perlite - Zeolite LTA (72hrs.) & 194 \\
Perlite - Zeolite LTA (96hrs.) & 246 \\
\hline
\end{tabular}

\subsection{Cd ion removal capability}

Fig. 6 shows the cadmium ion removal capability. It was confirmed that perlite has no cadmium ion removing ability. On the other hand, it was confirmed that zeolite surface modified perlite (perlite-zeolite) obtained by synthesizing on the surface of perlite has cadmium ion removing capability.

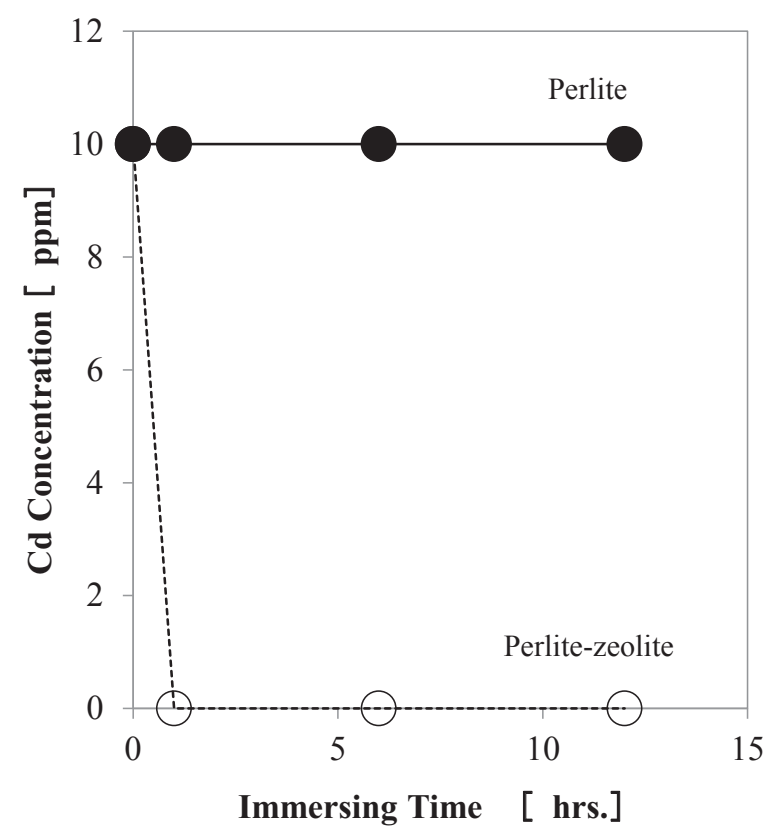

Fig.6 Relationship between $\mathrm{Cd}$ ion removal performance and immersing time of perlite and perlite-zeolite. 


\subsection{Characterization of the synthesized sample}

Fig.7 shows the K-edge XANES adsorption spectra of silicon. They were obtained in order to further examine the conditions of silicon in the derived specimen. Peaks of perlite, zeolite surface modified perlite (perlite-zeolite), as well as the four coordinate structure of silicon dioxide in the vicinity of $1847 \mathrm{eV}$, along with the LTA type zeolite reagent, were derived from Fig.7. A peak shift to lower energies was observed as the zeolite was synthesized from perlite, and the peak position was located between the perlite and LTA type zeolite reagent. A shoulder of the LTA type zeolite reagent was observed between $1850 \mathrm{eV}$ to $1853 \mathrm{eV}$, and a spectrum of perlite with LTA type zeolite on its surface was also observed. This spectrum had characteristics of perlite and the LTA type zeolite reagent, which suggest that the conditions of the electrons in the vicinity of the silicon atoms in perlite differed from the conditions of electrons in the LTA type zeolite. In addition, zeolite surface modified perlite had the electron conditions of the perlite and LTA type zeolite. Therefore, the spectrum had features of both the perlite and LTA type zeolite.

Fig. 8 shows the K-edge XANES spectra of aluminum. These spectra were used to further examine details of the conditions of aluminum in the derived specimen. Peaks of perlite, zeolite surface modified perlite (perlite-zeolite), as well as the four-coordinated structure of aluminium oxide in the vicinity of $1565 \mathrm{eV}$. A peak shift towards higher energies was observed when the zeolite was synthesized from perlite, and the peak position at 1565 $\mathrm{eV}$ matched with the position of LTA type zeolite. Furthermore, the results showed a significant difference between perlite and zeolite with regards to the spectra between $1568 \mathrm{eV}$ and $1580 \mathrm{eV}$. On the other hand, zeolite surface modified perlite showed a spectrum similar to that of the zeolite reagent. This indicates that the conditions of the electrons in the vicinity of the perlite aluminum atoms differ from the conditions of those in zeolite. Furthermore, since the zeolite surface modified perlite had electron conditions that were similar to those of LTA type zeolite, a prioritized structural change of aluminum in perlite is believed to have occurred when LTA type zeolite was generated from perlite to generate the LTA type zeolite.

Fig.9 shows the K-edge XANES spectra of cadmium. These spectra are the K-edge XANES spectra of cadmium ions adsorbed on zeolite surface modified perlite (Perlite-zeolite) and LTA type zeolite reagent. It was revealed that cadmium ions were adsorbed in divalent state to zeolite surface modified perlite (perlitezeolite) and LTA type zeolite reagent. Also, in the spectrum between $26730 \mathrm{eV}$ and $26750 \mathrm{eV}$, a difference was confirmed between the zeolite surface modified perlite and the LTA type zeolite reagent. It indicates the possibility that cadmium ions are adsorbed to the adsorption sites obtained on the surface of the perlite, which were treated during zeolite synthesis.

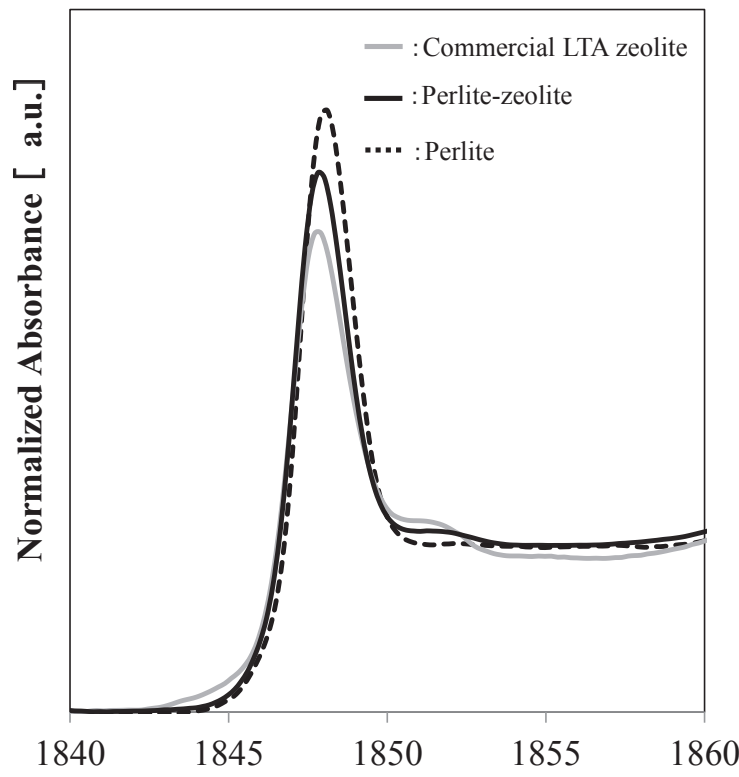

Photon Energy [ eV]

Fig.7 Si K-edge XANES spectra of perlite, perlite-zeolite, and LTA type zeolite reagent.

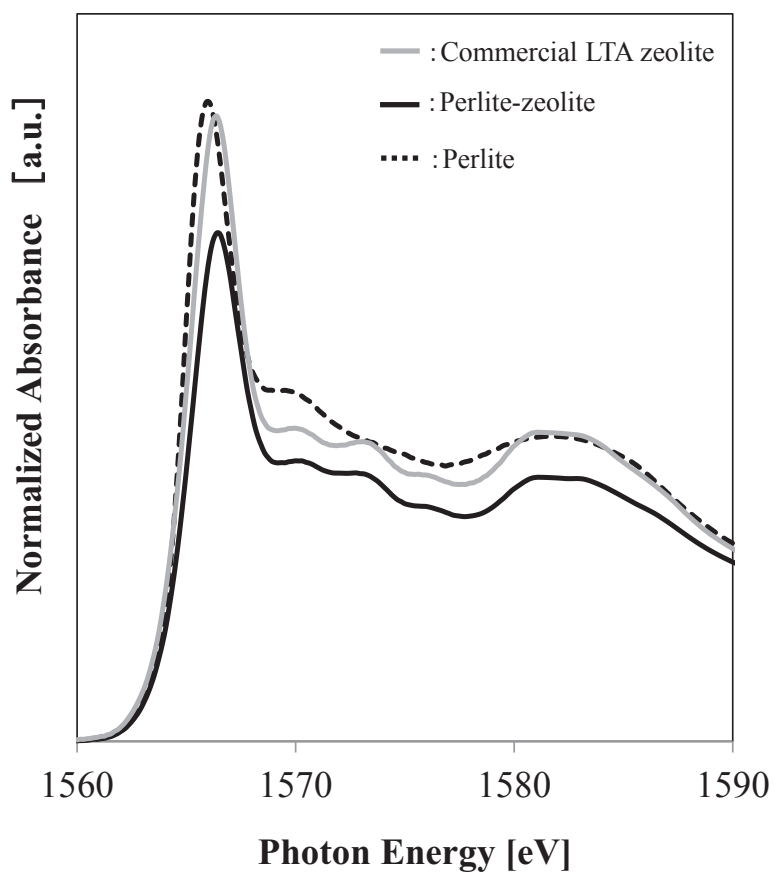

Fig.8 Al K-edge XANES spectra of perlite, perlite-zeolite, and LTA type zeolite reagent. 


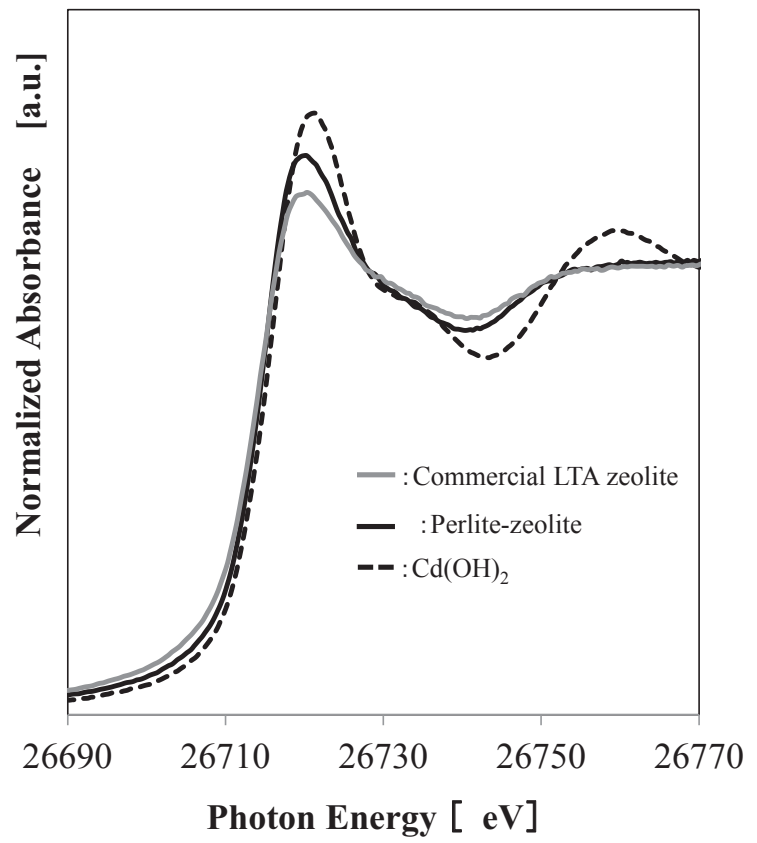

Fig.9 Cd K-edge XANES spectra of Cd absorbed perlitezeolite, and Cd absorbed LTA type zeolite reagent.

\section{Conclusions}

The aim of this work was to synthesize zeolite on the surface of perlite filter to improve its functionality, and the following results were obtained.

I. LTA type zeolite, with high crystallinity precipitates on the surface of perlite filter aid, was obtained through a hydrothermal synthesis using silicon to aluminum ratios of 2.0, or lower, and a reaction temperature of $55^{\circ} \mathrm{C}$.

II. A correlation was observed between the amount of precipitated zeolite and the reaction time. The amount of precipitated LTA type zeolite increased as the synthesis time increased.

III. Based on the SEM observations, it became evident that LTA type zeolite was precipitating from the surface of perlite.

IV. The results from the XAFS measurements also confirmed a structural change from perlite to LTA type zeolite based on the differences in the conditions of the electrons in the vicinity of silicon and aluminum, indicating that a portion of the perlite was converted into zeolite.

V. The results from the XAFS measurements of cadmium indicated the possibility that cadmium ions are adsorbed to the adsorption sites obtained on the surface of the perlite.

We succeeded in synthesizing LTA type zeolite on the surface of perlite filter aid, and derived perlite filter aid with added chemical characteristics that were not possible in the past. We intend to continue this work for the future employment of this material in environmental purification applications.

\section{Acknowledgments}

This research was conducted with the assistance provided by the Adaptable and Seamless Technology Transfer Program Through Target-driven R\&D (A-STEP) of the Japan Science and Technology Agency.

The light emission experiments of this research were conducted with the assistance of the "Photon Beam Platform Formation Project" of the Ministry of Education, Culture, Sports, Science and Technology, and were performed using the BL6N1 at the Aichi Synchrotron Radiation Center of the Aichi Science \& Technology Foundation (Experiment No: 2015PA003).

Furthermore, assistance was also provided by the "Advanced Research and Foundation Joint Platform Formation Project" of the Ministry of Education, Culture, Sports, Science and Technology, and the BL10 at the SR Center of Ritsumeikan University (Experiment No: R1517).

The synchrotron radiation experiments were performed at the BL14B2 of SPring-8 with the approval of the Japan Synchrotron Radiation Research Institute (JASRI) (Proposal No. 2015A1992, 2015B1595, and 2016A1540).

\section{References}

1. N. Koie, Introduction to ceramic materials and technologies, Industrial technology institute, 815-817 (1979).

2. K. Kimura, K. Hamano, Yogyo-kyokai-Shi, 84, 70-75 (1976)

3. N. Hara, H. Takahashi, Fundamentals and Applications of zeolite, Kodansha (1975)

4. S. Tangkawanit, K. Rangsriwatananon, Suranaree J. Sci. Technol, 12(1), 61-68 (2004)

5. P. Kongkachuichay and P. Lohsoontorn, Science Asia, 32, 13-16 (2006)

6. A. Dyer, S. Tangkawanit, K. Rangsriwatananon, Microporous and Mesoporous Materials, 75, 273-279 (2004),

7. A. Rujiwatra, Material Letters, 58, 2012-2015 (2004),

8. G.E. Christidis, I. Paspaliaris, A. Kontopoulos, Applied Clay Science, 15, 305-324 (1999) 